\title{
ciência
plurăl
}

\section{PERFIL EPIDEMIOLÓGICO DA MORTALIDADE NEONATAL NO RIO GRANDE DO NORTE-BRASIL: UM ESTUDO DE BASE SECUNDÁRIA}

\section{Epidemiological profile of neonatal mortality in Rio Grande do Norte-Brazil: a secondary based study}

Ana Mayara Gomes de Souza - Enfermeira, especialista em UTI neonatal pediátrica. Mestranda do Programa de Pós Graduação em Saúde Coletiva - PPGSCol da Universidade Federal do Rio Grande do Norte-UFRN. Bolsista Capes. E-mail: ana.mayara@yahoo.com.br

Talita Araújo de Souza - Enfermeira, especialista em urgência, emergência e UTI. Mestranda do PPGSCol-UFRN. Bolsista Capes. E-mail: talitaaraujo23@hotmail.com

Tainara Lôrena dos Santos Ferreira - Enfermeira, especialista em Auditoria em Saúde. Mestranda do PPGSCol-UFRN. E-mail: tainara_lorena@hotmail.com

Joycimara da Silva Sales de Medeiros - Enfermeira, especialista em UTI neonatal pediátrica pela Faculdade Metropolitana de Ciências e Tecnologias - CENPEX Cursos. E-mail: joycimara93@hotmail.com

Dandara Rayssa Silva de Souza - Enfermeira. Mestranda do PPGSCol-UFRN. E-mail: dandara_rayssa@hotmail.com

Fábia Barbosa de Andrade - Enfermeira. Doutora em Ciências da Saúde pela UFRN, Professora orientadora do PPGSCol-UFRN. E-mail: fabiabarbosabr@yahoo.com.br

Autor responsável pela correspondência:

Talita Araújo de Souza. E-mail: talitaaraujo23@hotmail.com 


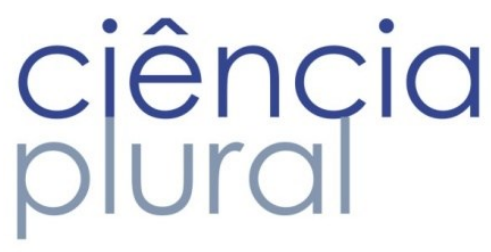

\section{RESUMO}

Introdução: Considerada um indicador da condição de vida e saúde da população, a mortalidade neonatal, responsável por $60 \%$ a $70 \%$ dos óbitos infantis nas últimas décadas ocorrem principalmente até $06^{\circ}$ dia de vida. Objetivo: Analisar o perfil epidemiológico da mortalidade neonatal no estado do Rio Grande do Norte numa série histórica de 2005 a 2014. Métodos: Estudo de série temporal e descritiva, com abordagem quantitativa baseado em dados secundários extraídos dos arquivos da Secretaria de Saúde do Rio Grande do Norte na $5^{\text {a }}$ Unidade Regional de Saúde Pública, sendo alguns dados complementares obtidos através do Sistema de Informação de Mortalidade (SIM) e Sistema de Informações sobre Nascidos Vivos (SINASC) do Departamento de Informática do Sistema Único de Saúde (DATASUS). Resultados: Os resultados demonstraram queda de 168 óbitos neonatais quando se compara o primeiro e o último ano avaliados. A média do coeficiente de mortalidade neonatal precoce e tardia diminuiu de 8,85 para 8,22, porém, ainda observa-se que neonatos do sexo masculino e de baixo peso tiveram aumento considerado chegando a $74,30 \%$ no último ano. Foram analisadas variáveis maternas e obstétricas onde se constatou que 0 número de óbitos neonatais de nascidos via cesariana veio crescendo principalmente entre mulheres mais jovens e de baixa instrução. Conclusão: Faz-se necessário um melhor acompanhamento e atenção dos gestores e serviços de saúde do Rio Grande do Norte, considerando os agravos epidemiológicos, bem como os outros fatores sociodemográficos inerentes ao processo saúde-doença dessa população, com vistas a promover uma redução significativa da mortalidade neonatal.

Palavras-chave: Mortalidade Infantil, Saúde da Criança, Sistemas de Informação.

\section{ABSTRACT}

Introduction: Considered an indicator of the condition of life and health of the population, neonatal mortality, responsible for $60 \%$ to $70 \%$ of infant deaths in the last decades mainly occurring up to the 6th day of life. Objective: To analyze the epidemiological profile of neonatal mortality in the state of Rio Grande do Norte in a historical series from 2005 to 2014. Methods: A descriptive and temporal series study with a quantitative approach based on secondary data extracted from the archives of the Health Department of Rio Grande do Norte in the 5th Regional Public Health Unit, some complementary data obtained through the Mortality Information System (SIM) and Information System on Live Births (SINASC) of the Department of Informatics of the Unified Health System (DATASUS). Results: The results showed a decrease of 168 neonatal deaths when the first and last year evaluated. The mean coefficient of early and late neonatal mortality decreased from 8.85 to 8.22 ; however, it is still observed that male and low birth weight infants had a considered increase reaching $74.30 \%$ in the last year. Maternal and obstetric variables were analyzed in which it was found that the number of neonatal deaths of those born via cesarean section increased mainly among younger and less educated women. Conclusion: It is necessary to better follow-up and care of health managers and services in Rio Grande do Norte, considering the epidemiological problems, as well as the other sociodemographic factors inherent in the health-disease process of this population, with a view to promoting a significant reduction of mortality neonatal.

Keywords: Child mortality, Child Health, Information systems. 


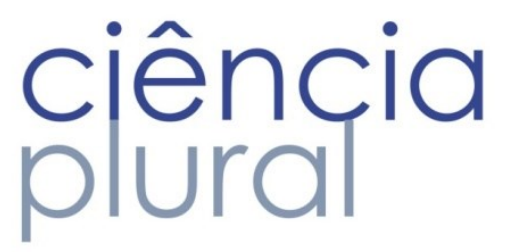

Introdução

A mortalidade neonatal se subdivide em dois componentes, aquela considerada neonatal precoce, quando se tem óbitos neonatais de 0 a 6 dias completos (primeira semana de vida), e a tardia, em que se consideram os óbitos ocorridos entre o sétimo e $027^{\circ}$ dia de vida. ${ }^{1,2}$ Apesar da redução nacional, a mortalidade neonatal ainda persiste como problema de saúde pública no Brasil e expressa desigualdades entre regiões e grupos sociais. ${ }^{3}$

Considerada um indicador da condição de vida e saúde da população, a mortalidade neonatal, responsável por $60 \%$ a $70 \%$ dos óbitos infantis nas últimas décadas ocorrem principalmente até o $6^{\circ}$ dia de vida e apresenta queda progressiva, em ritmo ainda aquém do desejado, requerendo mais esforços da sociedade, políticos e em especial da atuação dos profissionais envolvidos. ${ }^{4}$

A redução da mortalidade neonatal foi assumida como uma das metas para minimizar as desigualdades regionais no País em 2009 objetivou-se redução em até 5\% das taxas nas regiões da Amazônia Legal e do Nordeste brasileiro até o ano de 2010. A redução da mortalidade neonatal apresenta queda progressiva, como foi visto, mas os dados tornam-se preocupantes quando comparados aos países mais desenvolvidos que possuem taxa de duas a seis vezes menor. 5,6

Segundo os autores ${ }^{7}$ as condições epidemiológicas de redução da mortalidade infantil no Brasil e no mundo estão relacionadas à melhoria na organização do modelo de atenção à saúde da Estratégia Saúde da Família (ESF), à maior abrangência do saneamento básico, à realização de campanhas de vacinação, e principalmente aos progressos na qualidade da atenção à saúde da criança. Assim, a oferta e a qualidade da assistência à gestante durante o pré-natal e parto são determinantes para a promoção da saúde e prevenção da morbimortalidade da mãe e do recém-nascido $(\mathrm{RN}) .8$

As principais condições de risco para os recém-nascidos são o baixo peso (menores que $2500 \mathrm{~g}$ ), prematuridade (menores de 37 semanas), Apgar no $5^{\circ}$ minuto menor que 7, internamento, quando a mãe possui menos de 8 anos de escolaridade e quando é menor que 18 anos, sendo o período neonatal apontado como o principal risco para mortalidade infantil. ${ }^{4}$

Para enfrentarmos o desafio da redução dos óbitos infantis, o Sistema de Informação sobre Nascidos Vivos (Sinasc) e o Sistema de Informação sobre Mortalidade (SIM) têm sido importantes instrumentos de pesquisa, por serem bases de dados que possibilitam a detecção de fatores associados à mortalidade ${ }^{9}$. Os resultados obtidos nessas bases possibilitam um diagnóstico situacional e visualização da magnitude do problema. 
Considerando a mortalidade neonatal um indicador de saúde indispensável na avaliação da qualidade de saúde, bem como a escassez de estudos nessa área, o presente estudo objetivou analisar o perfil epidemiológico da mortalidade neonatal no estado do Rio Grande do Norte numa série histórica de 2005 a 2014, identificando em função dos dados apresentados, se houve aumento ou redução dos óbitos e as características associadas.

\section{MÉTODOS}

Trata-se de um estudo ecológico de série temporal e descritivo, baseado em dados secundários coletados a partir do Sistema de Informação sobre Mortalidade (SIM) e do Sistema de Informações sobre Nascidos Vivos (SINASC) no período de dez anos (2005 a 2014). Os dados foram obtidos por meio de solicitação oficial aos órgãos responsáveis pelos sistemas de informação, no caso a $5^{\text {a }}$ Unidade Regional de Saúde Pública ( $5^{\text {a }}$ URSAP) e os dados complementares foram obtidos através do acesso ao sítio oficial do Departamento de Informática do Sistema Único de Saúde (DATASUS), especialmente o SIM e SINASC.

Nos estudos ecológicos, compara-se a ocorrência da doença/condição relacionada à saúde e a exposição de interesse entre agregados de indivíduos (populações de países, regiões ou municípios, por exemplo) para verificar a possível existência de associação entre elas. ${ }^{10}$ Este tipo de pesquisa busca entender o problema utilizando registros de uma determinada população em determinado tempo e local específico.

Optou-se por utilizar os dados obtidos na primeira fonte citada, por serem mais consistentes, visto que foram colhidos mais próximos à fonte primária, sendo necessária a coleta dos demais dados na segunda fonte citada para a completa elucidação do objetivo deste.

Os dados foram organizados, armazenados e processados no software Statistical Package for the Social Science (SPSS), versão 22.0, com número de série: 10101141047, obtendo-se valores absolutos e relativos, médias e desvios padrão. Seguindo o objeto da investigação, foi avaliada a variável dependente, óbitos neonatais. Foram analisadas as seguintes variáveis independentes: nascidos vivos, coeficiente de mortalidade neonatal precoce, coeficiente de mortalidade neonatal tardia, sexo, baixo peso ao nascer, tipo de parto, anos de estudo materno e faixa etária materna.

Este estudo segue as normas de recomendações do Conselho Nacional de Saúde, referente à pesquisa com seres humanos. ${ }^{11}$ sendo dispensado da apreciação do Comitê de Ética em Pesquisa, por utilizar apenas dados secundários e de domínio público.

\section{RESULTADOS}


Os resultados sobre o número de óbitos neonatais por residência no RN, no período de 2005 a 2014, demonstram que este número oscilou ao longo do tempo, tendo apresentado uma queda quando se compara o primeiro e o último ano avaliados, com diminuição de 168 óbitos neonatais representando 3,8\% do total dos óbitos ocorridos no período, como observado no Gráfico 1.

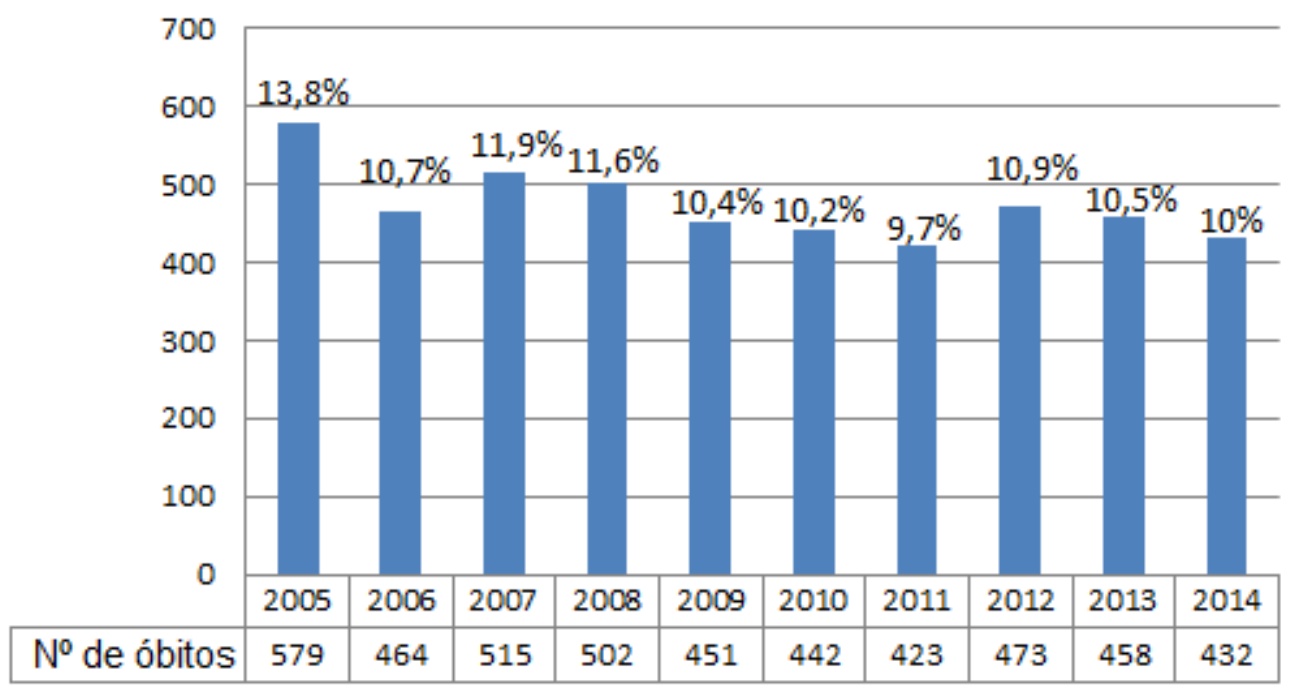

Gráfico 1 - Variação do número de óbitos neonatais por ano no período de 2005- 2014 no estado do Rio Grande do Norte. Brasil, 2017.

No que se refere à mortalidade neonatal, analisaram-se os dados considerando o período precoce e tardio. Nessa perspectiva, há uma prevalência dos óbitos neonatais precoces, sendo que o total destes, no período analisado, representou $80 \%$ dos óbitos neonatais, chegando a $84 \%$ no ano de 2014 , enquanto que os óbitos neonatais tardios tiveram o ápice no ano de 2012, representando $26 \%$ do total dos óbitos neonatais nesse ano, como observados na Tabela 1.

Ainda nesse sentido, observa-se que de acordo com a média do coeficiente de mortalidade, este variou ao longo do tempo, tanto para a mortalidade neonatal precoce (MNP) como para a tardia (MNT), prevalecendo à primeira em todos os anos analisados. Comparando-se o primeiro e o último ano estudados, a média do coeficiente de mortalidade precoce diminuiu de 8,85 para 8,22. Da mesma maneira, a média do coeficiente de mortalidade neonatal tardia diminuiu de 1,93 em 2005 para 1,47 em 2014, sendo que em 2012 esse coeficiente chegou a 3,19. Todos esses resultados por ano podem ser observados na tabela 1. 


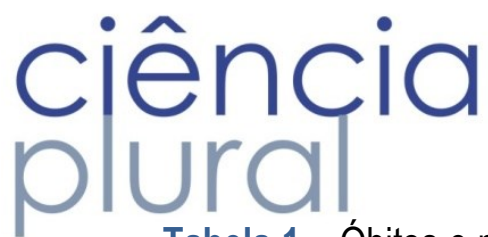

Tabela 1 - Óbitos e mortalidade neonatal por ano no período de 2005-2014 no estado do Rio Grande do Norte. Brasil, 2017.

\begin{tabular}{ccccccc}
\hline Ano & $\begin{array}{c}\text { Nascidos } \\
\text { vivos por } \\
\text { residência } \\
\mathrm{N}\end{array}$ & $\begin{array}{c}\text { Óbitos } \\
\text { neonatais } \\
\text { por } \\
\text { residência } \\
\mathbf{n}\end{array}$ & $\begin{array}{c}\text { Óbitos } \\
\text { neonatais } \\
\text { precoces } \\
\mathbf{n}(\%)\end{array}$ & $\begin{array}{c}\text { Óbitos } \\
\text { neonatais } \\
\text { tardios } \\
\mathbf{n}(\%)\end{array}$ & $\begin{array}{c}\text { Coeficiente } \\
\text { de MNP } \\
\text { media }\end{array}$ & $\begin{array}{c}\text { Coeficiente } \\
\text { de MNT } \\
\text { media }\end{array}$ \\
\hline $\mathbf{2 0 0 5}$ & 52.022 & 596 & $475(80 \%)$ & $121(20 \%)$ & 8,85 & 1,93 \\
$\mathbf{2 0 0 6}$ & 48.582 & 473 & $393(83 \%)$ & $80(17 \%)$ & 8,83 & 1,33 \\
$\mathbf{2 0 0 7}$ & 48.154 & 522 & $422(81 \%)$ & $100(19 \%)$ & 8,20 & 1,31 \\
$\mathbf{2 0 0 8}$ & 50.196 & 521 & $418(80 \%)$ & $103(20 \%)$ & 7,40 & 1,83 \\
$\mathbf{2 0 0 9}$ & 49.266 & 488 & $393(81 \%)$ & $95(19 \%)$ & 7,11 & 2,14 \\
$\mathbf{2 0 1 0}$ & 47.867 & 465 & $376(81 \%)$ & $89(19 \%)$ & 7,60 & 2,43 \\
$\mathbf{2 0 1 1}$ & 48.296 & 440 & $364(83 \%)$ & $76(17 \%)$ & 7,57 & 2,06 \\
$\mathbf{2 0 1 2}$ & 47.188 & 472 & $349(74 \%)$ & $123(26 \%)$ & 6,98 & 3,19 \\
$\mathbf{2 0 1 3}$ & 46.851 & 465 & $361(78 \%)$ & $104(22 \%)$ & 7,87 & 2,62 \\
$\mathbf{2 0 1 4}$ & 48.141 & 428 & $358(84 \%)$ & $70(16 \%)$ & 8,22 & 1,47 \\
Total & 486.563 & 4.870 & $3.909(80 \%)$ & $961(20 \%)$ & - & - \\
\hline
\end{tabular}

Foram observados o número de óbitos neonatais de nascidos com baixo peso, ou seja, com até $2.499 \mathrm{~g}$, constatou-se que a proporção de óbitos neonatais de crianças nascidas com baixo peso em relação ao total de óbitos neonatais, veio crescendo ao longo dos anos estudados, de modo que no primeiro ano estudado, $48,49 \%$ das crianças que foram a óbito no período neonatal, nasceram com baixo peso, enquanto que no ano de 2014 essa porcentagem chegou a 74,30\%, conforme demonstrado no Gráfico 2.

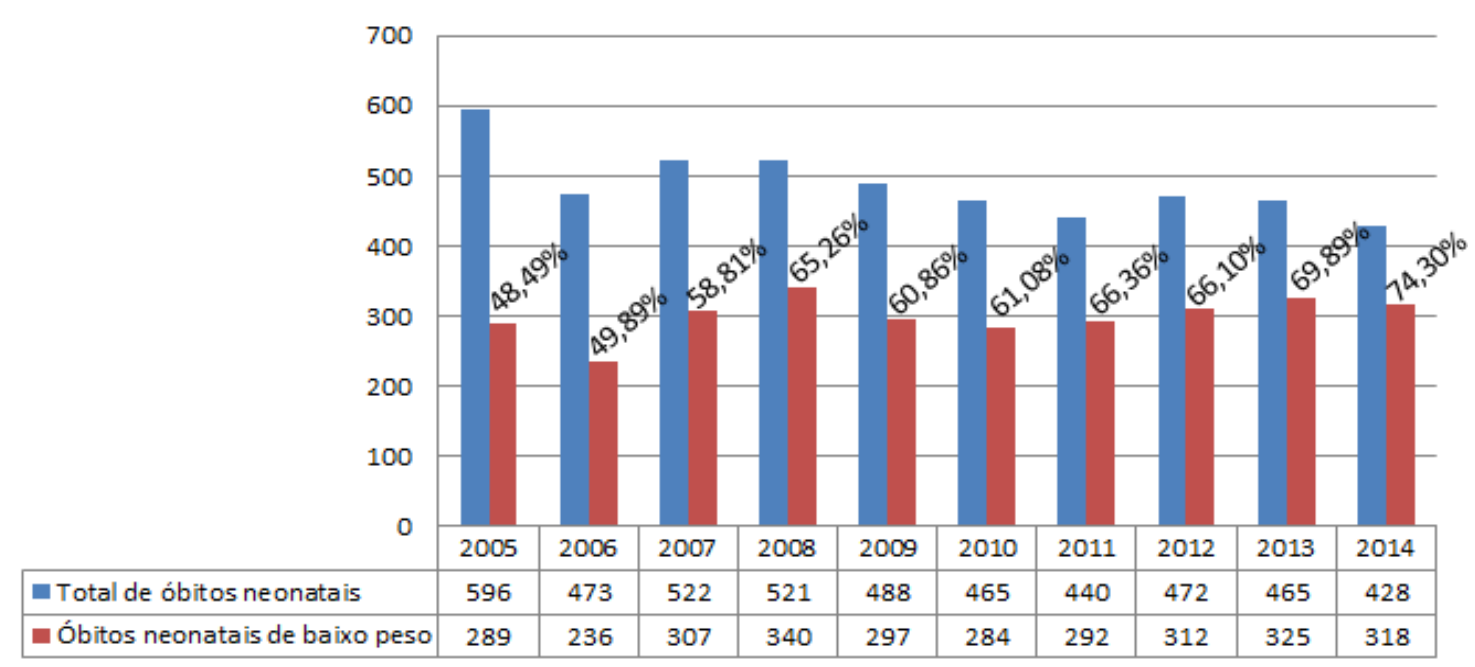

Gráfico 2 - Variação do número de óbitos neonatais de nascidos com baixo peso em relação ano número total de óbitos neonatais por ano no período de 2005-2014 no estado do Rio Grande do Norte. Brasil, 2017. Fonte: Dados do DATASUS. Adaptado (2018). 


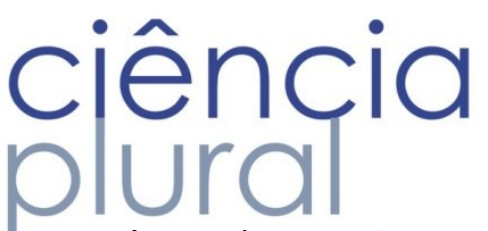

Também foi analisado o número de óbitos neonatais a partir do sexo. Obteve-se que o número de neonatos do sexo masculino que foram a óbito foi maior do que os do sexo feminino, mesmo se realizada a correção dos óbitos onde o sexo da criança foi ignorado, como observado no Gráfico 3.

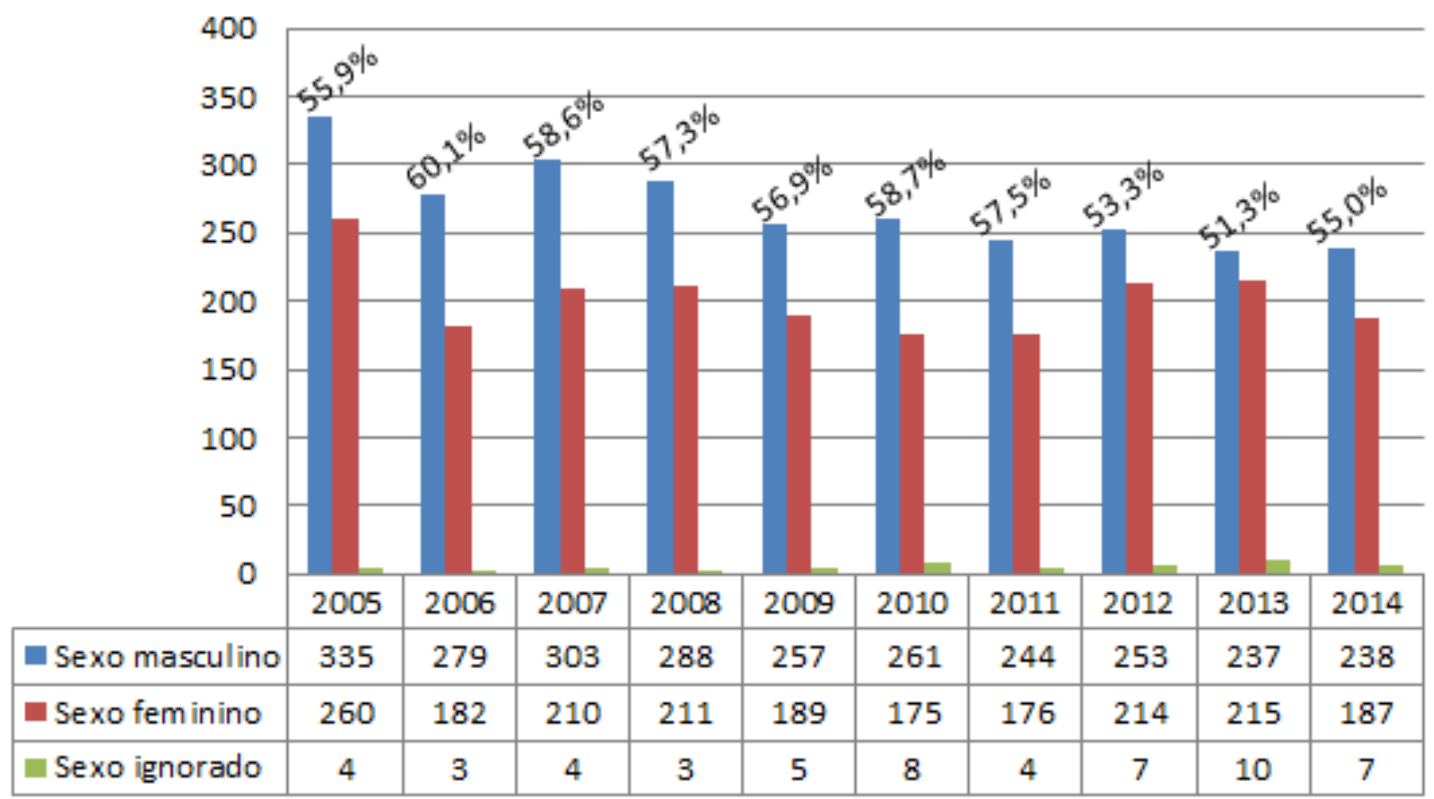

Gráfico 3 - Variação do número de óbitos neonatais conforme o sexo no período de 2005-2014 no estado do Rio Grande do Norte. Brasil, 2017. Fonte: Dados do DATASUS. Adaptado (2018).

Algumas variáveis maternas e obstétricas também foram consideradas. Nesse sentido, constataram-se que o número de óbitos neonatais de nascidos via cesariana veio crescendo ao longo do tempo, enquanto que por via vaginal esse número veio diminuindo. Sobre as variáveis maternas, destaca-se que o maior número de óbitos neonatais ocorreu de filhos de mães com idade entre 15 e 24 anos, em todos aqueles anos analisados. Nos dois primeiros anos, a maioria dos óbitos neonatais foi de nascidos de mães que estudaram de 4 a 7 anos, enquanto que a partir de 2007 a maioria desse número foi entre aquelas que tiveram entre 8 e 11 anos de estudo, conforme pode ser observado na integra e comparativamente na tabela 2. 


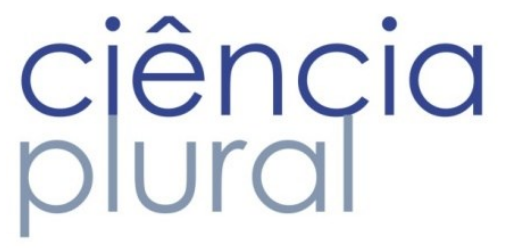

Tabela 2 - Número de óbitos neonatais por variáveis obstétricas e maternas no período de 2005-2014 no estado do Rio Grande do Norte. Brasil, 2017.

\begin{tabular}{ccccccccccc}
\hline Ano & $\mathbf{2 0 0 5}$ & $\mathbf{2 0 0 6}$ & $\mathbf{2 0 0 7}$ & $\mathbf{2 0 0 8}$ & $\mathbf{2 0 0 9}$ & $\mathbf{2 0 1 0}$ & $\mathbf{2 0 1 1}$ & $\mathbf{2 0 1 2}$ & $\mathbf{2 0 1 3}$ & $\mathbf{2 0 1 4}$ \\
\hline Tipo de parto & & & & & & & & & & \\
Cesariana & 133 & 114 & 135 & 175 & 156 & 182 & 166 & 190 & 209 & 200 \\
Vaginal & 297 & 247 & 302 & 287 & 258 & 222 & 218 & 224 & 217 & 195 \\
Ignorado & 169 & 103 & 80 & 40 & 37 & 40 & 40 & 60 & 36 & 37 \\
\hline Idade maternal & & & & & & & & & & \\
Até 14 anos & 3 & 9 & 8 & 12 & 9 & 8 & 6 & 10 & 11 & 7 \\
15 a 24 anos & 234 & 217 & 207 & 212 & 203 & 186 & 157 & 176 & 180 & 159 \\
25 a 34 anos & 128 & 115 & 152 & 164 & 137 & 134 & 140 & 148 & 154 & 150 \\
35 a 44 anos & 41 & 29 & 35 & 50 & 35 & 36 & 36 & 42 & 46 & 53 \\
44 a 64 anos & 1 & 1 & 1 & 2 & 3 & 3 & 1 & 1 & 1 & 1 \\
Ignorada & 192 & 93 & 114 & 64 & 65 & 77 & 85 & 95 & 70 & 62 \\
\hline Tempo de estudo materno & & & & & & & & & \\
1 a 3 anos & 42 & 36 & 31 & 29 & 24 & 18 & 32 & 44 & 26 & 19 \\
4 a 7 anos & 146 & 113 & 104 & 116 & 105 & 109 & 105 & 82 & 111 & 101 \\
8 a 11 anos & 112 & 97 & 125 & 140 & 146 & 111 & 114 & 146 & 144 & 127 \\
12 anos ou mais & 24 & 24 & 34 & 58 & 41 & 52 & 35 & 34 & 49 & 44 \\
$\quad$ Ignorado & 247 & 163 & 196 & 134 & 115 & 120 & 123 & 149 & 112 & 124 \\
\hline
\end{tabular}

\section{DISCUSSÃO}

O último censo demográfico realizado mostrou que esse declínio, entre os anos de 2000 e 2010, foi intenso na Região Nordeste, $58,6 \%{ }^{12}$ explicado principalmente pela mudança do padrão de fecundidade mais tardio (diminuição de $23,4 \%$ para essa região). Além disso, houve melhora no padrão de vida da população e políticas nas áreas de educação, saneamento básico e saúde, como o programa de saúde materno-infantil e a ampliação de serviços médico-hospitalares. Destacam-se ainda como obstáculos, as condições sociais e econômicas que interferem diretamente no acesso aos serviços e a informação. ${ }^{12,13}$

Foi possivel perceber essa redução, e os dados apresentaram oscilações importantes nos números de óbitos por residência e nos coeficientes de mortalidade neonatal (precoce e tardia). Essa redução, apesar de importante, não conseguiu atingir as metas propostas pelo país, elucidadas pela pactuação de 2009.

No Rio Grande do Norte ainda persiste uma progressão no número de óbitos neonatais precoces, expressos na Tabela 1. Contrapondo-se ao estudo em questão outros achados referentes ao país mostram que, dentre os estratos etários o componente pós-neonatal (28 dias a um ano de vida incompleto) sofreu 


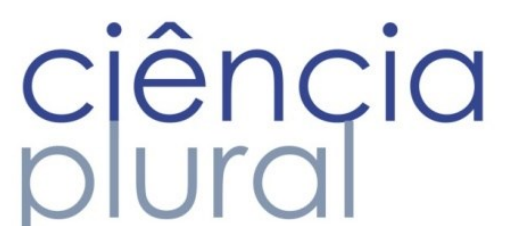

a maior queda, já o componente neonatal precoce ( 0 a 6 dias de vida) segue tendo a menor redução, assim como apresentado neste estudo. ${ }^{14}$

De modo geral, para o estado do Rio Grande do Norte, o controle de ações e mobilizações ainda deverá estar voltada a mortalidade neonatal (precoce e tardia), principalmente por ser a responsável por quase $70 \%$ das mortes no primeiro ano de vida. Nesse contexto, o cuidado adequado ao recém-nascido tem sido um dos desafios para reduzir os índices de mortalidade infantil em nosso país, passando a ter uma grande importância e suscitando ações para o seu controle, o que demanda mobilização e priorização na agenda dos gestores da saúde..$^{4,14}$

O componente neonatal da mortalidade infantil é aquele estreitamente vinculado aos cuidados no período da gestação, do nascimento e do recém-nascido, onde as principais falhas contribuem para 0 aumento da mortalidade fetal e neonatal precoce. ${ }^{14}$ Estima-se por um lado, que a maioria dos óbitos neonatais ocorra por causas evitáveis como infecção, asfixia ao nascer e complicações da prematuridade, por outro lado, mortes por pneumonia, diarreia e desnutrição persistem como causas importantes e evitáveis de mortes de crianças, incidindo principalmente no período pós-neonatal. ${ }^{14}$

Foi possível ainda a análise do peso ao nascer (Gráfico 2), considerando este como uma das características mais importantes do neonato, podemos observar que a tendência de mortalidade entre recém-nascidos (RN) de baixo peso teve valor substancial durante os dez anos analisados no estado em questão.

O baixo peso ao nascer $(<2.500 \mathrm{~g})$ é o fator de risco isolado mais importante para a mortalidade neonatal. ${ }^{14}$ Além disso, a detecção do peso dos recém nascidos pode atuar também sobre indicadores na fase infantil, o que pode se ressaltar que crianças com baixo peso apresentam uma maior mortalidade nas primeiras semanas de vida onde as taxas sobre determinado dado são variáveis nas diversas regiões do mundo, com evidentes desvantagens para os países menos desenvolvidos, pois estão associadas a condições socioeconômicas desfavoráveis e podem ser consideradas como um indicador de nível de saúde da população. ${ }^{15}$

Corroborando com os dados apresentados, estudo realizado no município de Serra, estado do Es pírito Santo observou que, quanto menor o peso maior é a chance de mortalidade neonatal, aqueles nascidos vivos com peso inferior a $1.500 \mathrm{~g}$ apresentaram risco de morrer no período neonatal 37,73 vezes maiores, no entanto, aqueles com peso de 1.500 a $2.499 \mathrm{~g}$, o risco passa a ser 4,56 vezes maior do que os nascidos vivos com peso superior a $2.500 \mathrm{~g} .{ }^{16}$

Outros fatores importantes são aqueles relacionados à mãe, como o seu estado nutricional antes e no período da gravidez, infecções geniturinárias, a primariedade, e até a questão da idade materna 


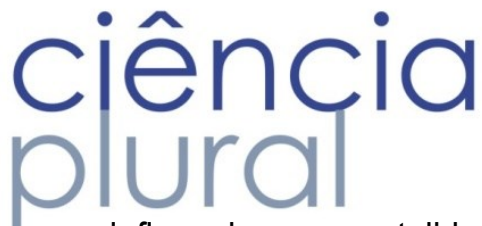

influenciam na mortalidade por baixo peso. ${ }^{16} \mathrm{Em} 2006$ cerca de 4\% das mães no Brasil foram consideradas desnutridas (IMC < 18,5), valor 33\% menor que o da década anterior. ${ }^{5}$

Outro ponto relevante é a correlação negativa entre baixo peso e prematuridade. Espera-se que RN com idade gestacional inferior a 37 semanas tenha peso menor que $2.500 \mathrm{~g}$, dessa forma, o controle da prematuridade deve ser política pública com o intuito de diminuir a proporção de baixo peso e suas consequências mais graves de morbimortalidade infantil. ${ }^{8}$

As práticas alimentares inadequadas nos primeiros anos de vida estão intimamente relacionadas à morbimortalidade de crianças, representada por doenças infecciosas, afecções respiratórias, cárie dental, desnutrição, excesso de peso, dentre outras. Uma das medidas primordiais para RN de baixo peso é a prática do incentivo ao aleitamento materno exclusivo, pois através do leite materno o neonato consegue ganhar peso adequadamente e manter-se protegido de comorbidades que podem por em risco sua sobrevivência, estimando-se que ações de promoção do aleitamento materno e de promoção da alimentação complementar saudável sejam capazes de diminuir, respectivamente, em até $13 \%$ e $6 \%$ à ocorrência de mortes em crianças menores de 5 anos em todo o mundo. ${ }^{3}$

Outrossim, é de suma importância orientações as gestantes quanto à adoção de uma alimentação adequada e saudável que supra suas necessidades nutricionais, garantindo um crescimento e desenvolvimento adequados ao feto. Dessa forma, os profissionais devem priorizar estratégias de educação nutricional e de promoção da alimentação saudável valorizando a cultura alimentar da gestante e/ou de sua família de forma a contribuir nesse processo. ${ }^{5}$

No Gráfico 3, é demonstrada a variação do número de óbitos neonatais a partir da variável sexo, onde observa-se que houve predominância de mortalidade para o sexo masculino em todos os anos estudados, corroborando com outros estudos: em que $68 \%$ dos casos analisados para o componente de mortalidade neonatal eram do sexo masculino ${ }^{17}$; onde $52,12 \%$ dos óbitos neonatais foram do sexo masculino ${ }^{18}$; onde $55,91 \%$ do total de óbitos no período analisado foram do sexo masculino ${ }^{19}$; onde $56,33 \%$ dos óbitos neonatais foram do sexo masculino2; foram $54,29 \%$ de óbitos neonatais do sexo masculino ${ }^{20}$.

Portanto, constata-se que em diversos contextos, mais da metade dos óbitos neonatais são de crianças do sexo masculino. Segundo autores ${ }^{21}$, nos recém-nascidos do sexo masculino o amadurecimento do sistema respiratório ocorre de forma mais tardia quando comparados ao do sexo feminino e, por isto, aqueles estão mais vulneráveis a problemas respiratórios, uma das principais causas de óbito no período neonatal.

$\mathrm{Na}$ Tabela 2 foram descritos os fatores maternos e obstétricos que influenciaram na variação dos óbitos neonatais no Rio Grande do Norte. Os óbitos de crianças por partos cesáreos aumentaram ao longo 


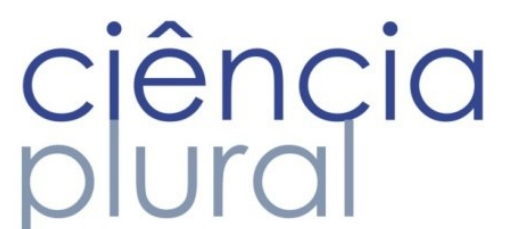

dos anos estudados, e os nascidos por parto vaginal à minoria. Sobre essa variável, pode-se inferir que a cesariana vem se mostrando como um fator de risco para a ocorrência de óbitos neonatais devido à complexidade exigida por este.

A Organização Mundial da Saúde estabelece que apenas 15\% dos partos necessitariam ser operatórios, porém, infelizmente no Brasil quase metade dos partos são cesarianas, que em sua maioria são realizadas desnecessariamente quando na verdade só deveriam ocorrer quando houvesse risco para a mãe ou para o bebê. Em geral, todo parto pode ser por via vaginal e natural (ou humanizado). 0 parto normal é a maneira mais segura e saudável de ter filhos e, por isso, deve ser estimulado por meio de uma assistência humanizada, segura e de qualidade, além do mais, as gestantes precisam reivindicar seu direito ao parto normal e serem informadas sobre as razões que a impedem de optar por este 22 .

A escolaridade materna é outro fator de risco importante para a sobrevivência infantil e indicador da condição socioeconômica ${ }^{4}$. A idade materna é considerada uma variável importante na determinação da mortalidade neonatal, pois os extremos de idade para concepção apresentam maiores chances de complicações e consequente risco de morte ${ }^{16}$.

Segundo dados do Ministério da Saúde 40\% das mães em 2008 tinham menos de 8 anos de instrução, variando entre $39 \%$ na região Nordeste, novamente demonstrando as desigualdades sociais existentes no País ${ }^{4}$. Em estudo realizado no município de Salvador (Bahia), confirmou-se que a proporção de óbitos foi maior entre crianças de mães com menor instrução, sendo de apenas 10,62\% entre aqueles de mulheres com 12 ou mais anos de estudo22.

Os autores reforçam que a forte associação da mortalidade neonatal precoce com a baixa instrução materna indica que as gestantes com menor escolaridade devem ser alvo de atenção especial nos serviços de saúde, independente de qualquer outra característica que possam apresentar, entende-se que o número maior de consultas no pré-natal é um fator protetor relevante para o óbito neonatal, indicando que a importância dos cuidados durante a gestação, assim como, um acompanhamento gestacional mais assíduo pode identificar precocemente e prevenir ocorrências lesivas para o recém-nascido22.

Por fim, ao analisar a variável escolaridade maternal relacionada à quantidade de óbitos neonatais, observa-se que o maior número de óbitos desse tipo ocorreu de filhos de mulher que tinham de 4 a 7 e de 8 a 11 anos de estudo, dando ênfase ao número de casos com informações ignoradas, o que poderia modificar sensivelmente os valores, casos tivessem sido adequadamente preenchidos.

Estima-se que $20 \%$ de todos os nascidos vivos no Brasil sejam de mães adolescentes apresentando maiores fatores de risco para a mortalidade fetal e infantil como prematuridade e baixo peso ao nascer, $20 \%$ de todas as internações por gravidez, parto, aborto ou puerpério estão dentro da faixa etária dos 10 


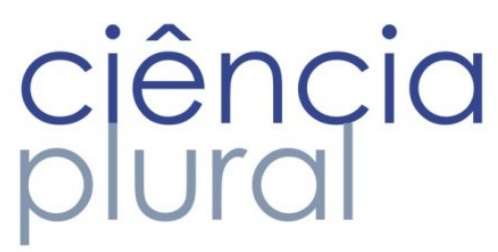

aos 19 anos. Torna-se necessário encarar a questão da maternidade na adolescência como problema de saúde pública. Para isso é importante à implantação de efetivas políticas sociais e de saúde voltadas aos adolescentes reestruturando a atenção ao pré-natal e ao parto ${ }^{8}$.

\section{CONCLUSÃO}

Foi possivel identificar redução da mortalidade, apesar da oscilação dos dados encontrados, necessitando de melhor acompanhamento dos gestores e serviços de saúde. Recomenda-se a análise de outras variáveis de interesse para esta temática, como o Apgar, a prematuridade, o tempo de internação do binômio mãe-bebê, a instituição de internamento (pública ou privada) e por fim a renda e estado civil da mãe, entre outras.

Reconhece-se como fragilidade intrínseca a este estudo, tratar-se da análise de dados secundários, conhecendo-se a qualidade insatisfatória e a incompletude dos dados nos bancos oficiais. Apesar disso, esses resultados poderão contribuir significativamente para reflexão e debate sobre a temática, dada a sua importância como problema de saúde pública.

\section{REFERÊNCIAS}

1. Paula Júnior JD, Lucas ES, Cunha LMC, Machado MGM, Pedrosa RL. Perfil da mortalidade neonatal no município de Ubá/MG, Brasil (2008-2010). Rev. Bras. Pesq. Saúde. 2016; 18(3): 24-31.

2. Araújo Filho CAA, Sales IMM, Araújo AKL, Almeida PD, Rocha SS. Aspectos epidemiológicos da mortalidade neonatal em capital do nordeste do Brasil. Rev Cuid. 2017; 8(3): 1767-76.

3. Brasil. Ministério da Saúde. Secretaria de Atenção à Saúde. Departamento de Atenção Básica. Manual instrutivo das ações de alimentação e nutrição na Rede Cegonha. Brasília (DF). 2013.

4. Brasil. Ministério da Saúde. Secretaria de Atenção à Saúde. Departamento de Ações Programáticas e Estratégicas. Atenção à saúde do recém-nascido: guia para os profissionais de saúde. Brasília (DF). 2011.

5. Brasil. Ministério da Saúde. Secretaria de Ciência, Tecnologia e Insumos Estratégicos. Departamento de Ciência e Tecnologia. Avaliação da atenção ao pré-natal, ao parto e aos menores de um ano na Amazônia Legal e no Nordeste, Brasil, 2010. Ministério da Saúde - Brasília (DF). 2013.

6. Fundo das Nações Unidas para a Infância (UNICEF). Committing to Child Survival: a Promise Renewed Progress Report 2013. New York, 2013. 


\section{ciência \\ plural}

7. Kropiwiec MV, Franco SC, Amaral AR. Fatores associados à mortalidade infantil em município com índice de Desenvolvimento humano elevado. Rev Paul Pediat. 2017.

8. Melo EC, Oliveira RR, Nonaka RH, Mathias TAF. Fatores relacionados ao parto cesáreo, baixa cobertura de pré-natal e baixo peso ao nascer. REAS [Internet]. 2013; 2(1):47-59.

9. Sanders LSC et al. Mortalidade infantil: análise de fatores associados em uma capital do Nordeste brasileiro. Cad. Saúde Colet. 2017; 25 (1): 83-89.

10. Lima CMF, Barreto SM. Tipos de estudos epidemiológicos: conceitos básicos e aplicações na área do envelhecimento. Epidemiol. serv. saúde. 2003; 12(4): 189-201.

11. Conselho Nacional de Saúde. Resolução No 510/2016 - Ética na Pesquisa na área de Ciências Humanas e Sociais: conquista dos pesquisadores. 2016. Acessado em: 15 de dez 2017.

12. Instituto Brasileiro de Geografia e Estatística. Evolução e perspectivas da mortalidade infantil no Brasil. Rio de Janeiro, 1999.

13. Instituto Brasileiro de Geografia e Estatística. Censo Demográfico 2010: Rio de Janeiro. Brasília, 2012.

14. Brasil. Ministério da Saúde. Secretaria de Ciência, Tecnologia. Secretaria de Ciência, Tecnologia e Insumos Estratégicos. Departamento de Ciência e Tecnologia. Síntese de evidências para políticas de saúde: mortalidade perinatal. 2. ed. - Brasília (DF). 2016.

15. Galvão KB, Araújo MSJL, Vasconcelos ESPRB, Diniz MSGMD, Nascimento EGC. Assistência a saúde do recém-nascido com baixo peso. Revista da Universidade Vale do Rio Verde, Três Corações. 2015; 13(2): 251-264.

16. Lima EFA, Sousa Al, Griep RH, Primo CC. Fatores de risco para mortalidade neonatal no município de Serra, espírito Santo. Rev Bras Enferm. 2012; 65(4): 578-85.

17. Santos EP, Ferrari RAP, Bertolozzi MR, Cardelli AAM, Godoy CB, Genovesi FF. Mortalidade entre menores de um ano: análise dos casos após alta das maternidades. Rev Esc Enferm USP. 2016; 50(3): 390-398.

18. Demitto MO, Gravena AAF, Dell'Agnolo CM, Antunes MB, Pelloso SM. High risk pregnancies and factors associated with neonatal death. Rev Esc Enferm USP. 2017; 51(3): 208-214.

19. Moreira KFA, Bicalho BO, Santos LCS, Amaral FMGS, Órfão NH, Cunha MPL. Perfil e evitabilidade de óbito neonatal em um município da amazônia legal. Cogitare enferm. 2017; 22(2): 01-10.

20. Lima MM, Aguilar AMM. Análise dos Indicadores de Saúde Materno Infantil de um Município do Estado do Mato Grosso. J. health sci. 2017; 19(3): 183-0. 


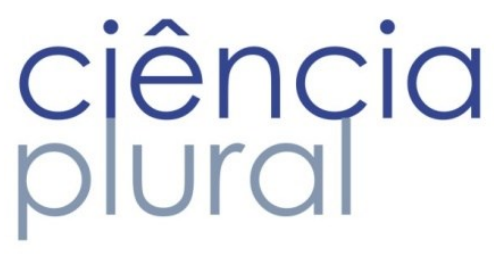

21. Brasil. Ministério da Saúde. Manual dos comitês de prevenção do óbito infantil e fetal. Brasil. Brasília (DF). 2005.

22. Guia dos Direitos da Gestante e do Bebê / UNICEF - Fundo das Nações Unidas para a Infância; [ilustrações de Ziraldo]. -- São Paulo: Globo, 2011.

Submetido: $10 / 07 / 2018$

Aprovado: 02/02/2019 